\title{
Antimicrobial resistance in bacteria
}

Review Article

\author{
Katrijn Bockstael ${ }^{*}$, Arthur Van Aerschot ${ }^{* * *}$ \\ Laboratory for Medicinal Chemistry, Rega Institute for Medical Research, \\ Katholieke Universiteit Leuven, 3000 Leuven, Belgium
}

Received 9 July 2008; Accepted 17 November 2008

\begin{abstract}
The development of antimicrobial resistance by bacteria is inevitable and is considered as a major problem in the treatment of bacterial infections in the hospital and in the community. Despite efforts to develop new therapeutics that interact with new targets, resistance has been reported even to these agents. In this review, an overview is given of the many therapeutic possibilities that exist for treatment of bacterial infections and how bacteria become resistant to these therapeutics.

Keywords: Antimicrobial agents • Resistance development • Efflux • Alteration of drug target $\bullet$ Antibacterials

(c) Versita Warsaw and Springer-Verlag Berlin Heidelberg.
\end{abstract}

\section{Introduction}

The history of humankind can be regarded from a medical point of view as a struggle against infectious diseases. Infections were the leading cause of death worldwide at the beginning of the $20^{\text {th }}$ century. Since the discovery of penicillin by Alexander Fleming in 1929 and the first introduction of the sulpha drugs by Domagk in 1932, the number of new antimicrobials available has increased tremendously between 1940 and 1960. 'The era of antibiotics' led to optimism till the early 1970s that infectious diseases can be controlled and prevented and mankind felt confident that modern medicine would prevail. However, infections are still the second-leading cause of death worldwide, causing over 13 million deaths each year. This fact is the result of the emergence of new diseases, the re-emergence of diseases once controlled and more specifically of the development of antimicrobial resistance $[1,2]$.

Bacteria have a remarkable ability to adapt to adverse environmental conditions, which is an example of the ancient law of nature of 'survival of the fittest'. It appears that the emergence of antimicrobial resistant bacteria is inevitable to most every new drug and it is recognized as a major problem in the treatment of microbial infections in hospitals and in the community [2].

\footnotetext{
*E-mail: Katrijn.Bockstael@rega.kuleuven.be

**E-mail: Arthur.VanAerschot@rega.kuleuven.be
}

\section{Different mechanisms of resistance to antimicrobials}

\subsection{Intrinsic resistance}

Bacteria may be inherently resistant to an antimicrobial. This passive resistance is a consequence of general adaptive processes that are not necessary linked to a given class of antimicrobials. An example of natural resistance is Pseudomonas aeruginosa, whose low membrane permeability is likely to be a main reason for its innate resistance to many antimicrobials [2]. Other examples are the presence of genes affording resistance to self-produced antibiotics, the outer membrane of Gram-negative bacteria, absence of an uptake transport system for the antimicrobial or general absence of the target or reaction hit by the antimicrobial [3].

\subsection{Acquired resistance}

Active resistance, the major mechanism of antimicrobial resistance, is the result of a specific evolutionary pressure to develop a counterattack mechanism against an antimicrobial or class of antimicrobials so that bacterial populations previously sensitive to antimicrobials become resistant [3]. This type of resistance results from changes in the bacterial genome. Resistance in bacteria may be acquired by a mutation and passed vertically by selection to daughter cells. More commonly, resistance is acquired by horizontal transfer of resistance genes 
Figure 1. Main mechanisms of active antimicrobial resistance.

Decreasing uptake or increasing efflux

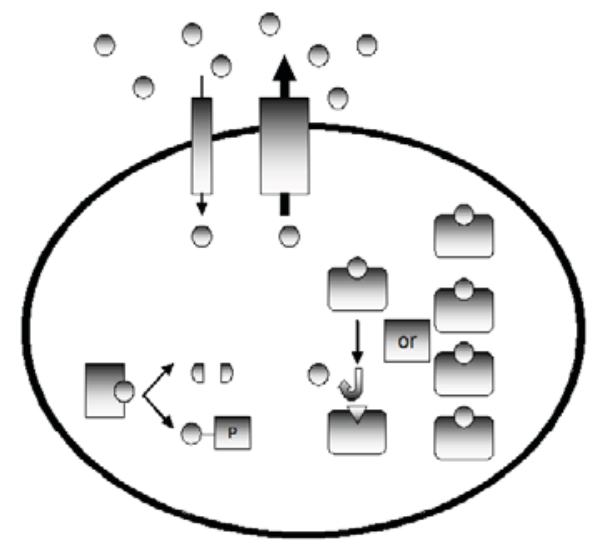

Antibiotic inactivation

Drug target alteration

$$
\begin{aligned}
& \text { - Hydrolysis } \\
& \text { - Group transfer } \\
& \text { - Other enzymatic mechanisms }
\end{aligned}
$$

between strains and species. Exchange of genes is possible by transformation, transduction or conjugation [4].

The major mechanisms of active antimicrobial resistance (Figure 1) are (1) prevention of accumulation of antimicrobials either by decreasing uptake or increasing efflux of the antimicrobial from the cell via a collection of membrane-associated pumping proteins, (2) qualitative drug target site alteration, which reduces the affinity for antimicrobials either by mutation or by target modification, or quantitative drug target alteration by overproduction of the target and (3) inactivation of antibiotics either by hydrolysis or by modification $[2,3]$.

\subsubsection{Prevention of antimicrobial access to their targets}

Permeability barriers

The cytoplasmic membrane is a barrier to hydrophilic compounds. Entry of cytoplasmatically targeted compounds is usually through carrier-mediated transport mechanisms or via channels in the outer membrane of Gram-negative bacteria formed by porins (e.g. OprD porin). Antibacterial compounds transported in this way may be subject to resistance by loss of non-essential transporters, by lack of porins or by mutations that are able to modify the structure of these channels and thus decreasing the influx $[2,5]$. Some microbes possess impermeable cell membranes that prevent drug influx as exemplified by $P$. aeruginosa. Furthermore, many large molecule antimicrobials are naturally inactive against certain groups of bacteria because they simply can not pass into the bacterial cell [4].
Efflux pumps

Increasing the efflux also plays a role, especially with hydrophobic compounds that presumably enter the cell via diffusion [5]. At the same speed where these antimicrobials are entering the cell, efflux mechanisms are pumping them out again, before they reach their target [6]. A mutation resulting in overexpression of a multidrug efflux pump leads to resistance to a wide variety of structurally unrelated antimicrobials [2]. Multidrug resistance proteins (MDRs) or multidrug efflux pumps are widespread in bacteria [7]. They are grouped into five families based on their mechanisms and primary sequence homologies. The major facilitator superfamily (MFS), the resistance-nodulation-division (RND) family, the small multidrug resistance (SMR) family and the multidrug and toxic compounds extrusion (MATE) family are secondary transporters using either proton motive force (PMF) or sodium ion motive force (only for the MATE proteins) to expel antimicrobials from cells. Members of the ATP-binding cassette (ABC) superfamily are primary transporters using energy liberated by ATP hydrolysis [2].

\subsubsection{Alteration of drug target}

Natural variations or acquired changes in the target sites of antimicrobials that prevent drug binding or action is a common mechanism of resistance. Target site changes often result from spontaneous mutation of a bacterial gene on the chromosome and selection in the presence of the antimicrobial $[4,8]$.

\subsubsection{Antibiotic inactivation}

Some bacteria produce modifying enzymes that reside within or near the cell surface, which selectively target and inactivate the drug. Enzymatic inactivation either by hydrolysis or by modification (group transfer and redox mechanisms) is a major mechanism of resistance to natural antibiotics in pathogenic bacteria [2]. The resistant isolates in most cases inherit the antibioticresistance genes on resistance $(R)$ plasmids. These resistance determinants are most probably acquired by pathogenic bacteria from a pool of resistance genes in other microbial genera, including antibiotic producing organisms. No enzymes that hydrolyse or modify manmade antimicrobials have been found [2]. Furthermore, antibiotic inactivation mechanisms share many similarities with well-characterized enzymatic reactions and resistance proteins show homologies to known metabolic and signalling enzymes with no antibiotic resistance activity. Therefore, one can speculate that these are the original sources of resistance [3].

Either hydrolysis or group transfer reactions, or alternatively oxidation or reduction reactions, can sign for 
Figure 2. Actually used targets for antimicrobial agents.

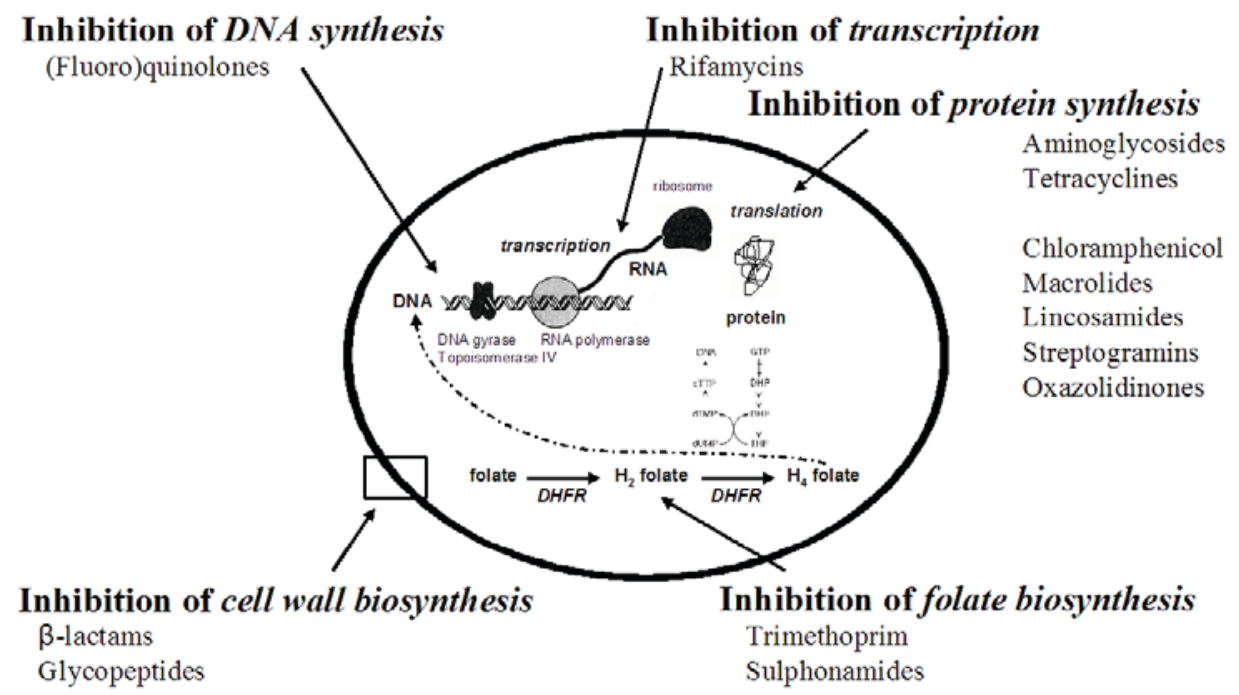

the inactivation mechanism. Many antibiotics possess hydrolytically susceptible chemical bonds (e.g. esters and amides) whose integrity is central to biological activity. When these vulnerable bonds are cleaved, the antibiotic activity is destroyed [3]. The most diverse and largest family of resistance enzymes is the group transferases. Those enzymes covalently modify antibiotics leading to structural alterations that impair target binding. Chemical strategies include $\mathrm{O}$-acylation and $\mathrm{N}$-acylation, O-phosphorylation, O-nucleotidylation, O-ribosylation, O-glycosylation and thiol transfer [3]. The oxidation or reduction of antibiotics has not been frequently exploited by pathogenic bacteria. Lyases are enzymes that cleave $\mathrm{C}-\mathrm{C}, \mathrm{C}-\mathrm{O}, \mathrm{C}-\mathrm{N}$ and $\mathrm{C}-\mathrm{S}$ bonds by non-hydrolytic or non-oxidative routes. These reactions frequently result in double bond formation or ring closure [3].

\section{Selected antimicrobial agents according to mechanisms of action}

A key feature of the target sites for antimicrobial agents is their vital role in microbial growth and survival [8]. Antimicrobials are usually classified on the basis of their mode of action. The main classes of antimicrobials inhibit four classical targets (Figure 2): (1) cell wall biosynthesis, (2) protein biosynthesis, (3) nucleid acid biosynthesis and (4) folate biosynthesis [2,6]. Structures of some representatives for each discussed antimicrobial class are given in Figure 3. In Table 1, an overview of the targets used by commercialised antimicrobial agents is given. Table 2 summarises the resistance mechanisms to the main antimicrobial classes.

\subsection{Inhibitors of bacterial cell wall biosynthesis}

Bacterial cells are surrounded by a cell wall or layers of peptidoglycan. This is a mesh-like carbohydrate polymer, which provides the mechanical support necessary to protect themselves from osmolysis [9]. Peptidoglycan is composed of linear chains of $\beta-(1,4)$ $N$-acetyl hexosamine units joined by peptide cross-links. The peptidoglycan undergoes cross-linking of the glycan strands by the action of transglycosidases and of the peptide strands by the action of transpeptidases, also called penicillin-binding proteins (PBPs) [10]. Inhibitors of one of both enzymes, active in the last stage of cell wall biosynthesis, fall into two major classes with respect to their mechanism of action, the $\beta$-lactams and the glycopeptides (Figure 4).

\subsection{1. $\beta$-lactam antibiotics}

The primary targets of the $\beta$-lactam agents are the PBPs. Upon nucleophilic attack on the $\beta$-lactam ring by the side chain oxygen atom of a serine residue at the active site of the enzyme, a relatively stable lethal covalent penicilloyl-enzyme complex is formed in which the serine is covalently acylated by the hydrolysed $\beta$-lactam, leading to inactivation of the enzyme and blocking of the normal transpeptidation reaction $[2,8,9,11]$. This results in weakly cross-linked peptidoglycan and eventually cell lysis and death $[6,11]$.

\section{Antibiotic inactivation}

$\beta$-Lactamases are hydrolytic enzymes that disrupt the amide bond of the characteristic $\beta$-lactam ring, before the antibiotic can get to the site of cell wall synthesis, rendering the antimicrobial ineffective [12]. $\beta$-Lactamase 
Figure 3. Structures of some representatives for the discussed antimicrobial classes.

$$
\beta \text {-lactams }
$$

e.g. penicillin<smiles>[R]C(=O)N[C@H]1C(=O)N2[C@@H]1SC(C)(C)[C@H]2C(=O)O</smiles>

Rifamycins

e.g. rifampicin

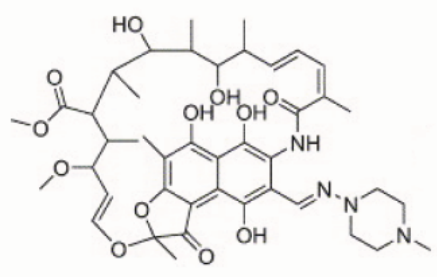

Chloramphenicol<smiles>O=C(NC(CO)C(O)c1ccc([N+](=O)[O-])cc1)C(Cl)Cl</smiles>

Streptogramins A

e.g. dalfopristin<smiles>CCN(CC)CCS(=O)(=O)C1CCN(C(=O)c2coc(CC(=O)CC(O)C=C(C)C=CCNC(=O)C=C[C@@H](C)C(O)C(C)C)n2)C1C(=O)O</smiles>

Sulphonamides

e.g. sulphamethoxazole<smiles>Cc1cc(NS(=O)(=O)c2ccc(N)cc2)no1</smiles>

Glycopeptides

e.g. vancomycin

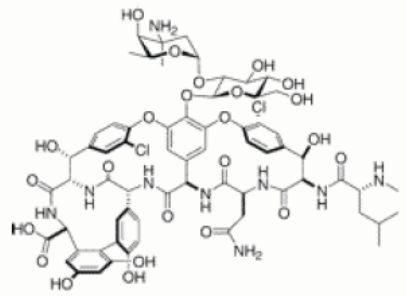

Aminoglycosides

e.g. streptomycin

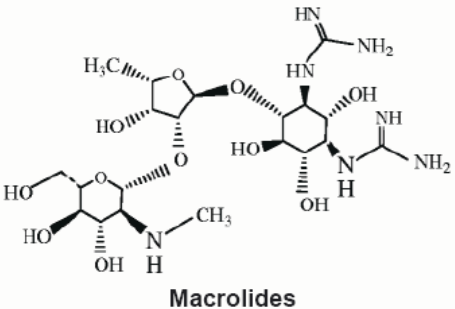

e.g. erythromycin

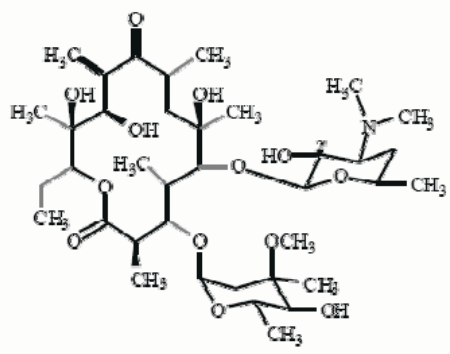

Streptogramins B

e.g. quinupristin

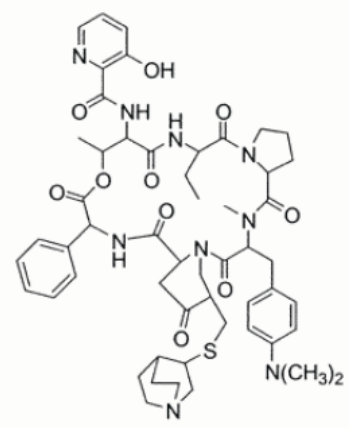

Trimethoprim<smiles>COc1cc(Cc2cnc(N)nc2N)cc(OC)c1OC</smiles>

(Fluoro)quinolones

e.g. ciprofloxacin<smiles>[Z]c1cc2c(=O)c(C(=O)O)cn(C3CC3)c2cc1N1CC[Pb]CC1</smiles>

Tetracyclines

e.g. doxycycline

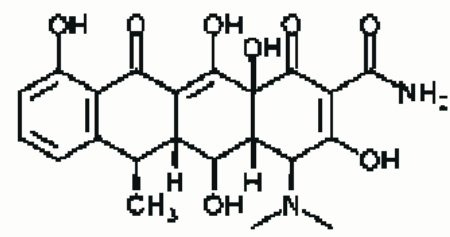

Lincosamides

e.g. lincomycin

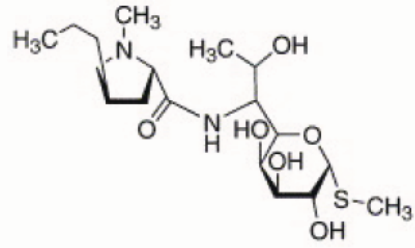

Oxazolidinones

e.g. linezolid<smiles>CC(=O)NCC1CN(c2ccc(N3CCOCC3)c(F)c2)C(=O)O1</smiles>

Daptomycin

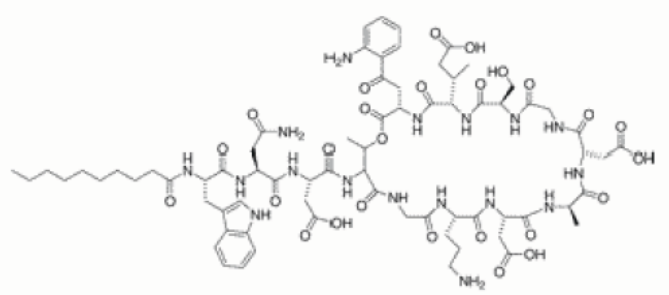


Table 1. Targets used by commercialised antimicrobial agents (adapted from reference 38). Antimicrobial classes highlighted in bold are discussed.

\begin{tabular}{|c|c|c|c|}
\hline Target & Antibacterial class & Example & Principle target \\
\hline \multirow[t]{12}{*}{ Cell wall biosynthesis } & \multirow[t]{3}{*}{$\beta$-lactams } & Penicillin & \multirow[t]{3}{*}{ PBPs (transpeptidases) } \\
\hline & & Ampicillin & \\
\hline & & Methicillin & \\
\hline & $\beta$-lactamase-inhibitors & Clavulanic acid & $\beta$-lactamase \\
\hline & \multirow[t]{2}{*}{ Glycopeptides } & Vancomycin & \multirow[t]{2}{*}{ Terminal D-Ala-D-Ala in Lipid II } \\
\hline & & Teicoplanin & \\
\hline & Ramoplanin & & Terminal D-Ala-D-Ala in Lipid II \\
\hline & Telavancin & & Terminal D-Ala-D-Ala in Lipid II \\
\hline & Cyclic peptides & Bacitracin & Undecaprenyl \\
\hline & \multirow[t]{2}{*}{ Amino acid analogue } & \multirow[t]{3}{*}{ D-cycloserine } & Alanine racemase \\
\hline & & & D-Ala-D-Ala ligase \\
\hline & Fosfomycin & & MurA1, MurA2 \\
\hline \multirow[t]{5}{*}{ Bacterial cell membranes } & $\begin{array}{l}\text { Cationic peptides } \\
\text { polymyxins }\end{array}$ & Colistin & Lipopolysaccharides in outer membrane \\
\hline & Lipopeptides & Daptomycin & Cytoplasmic membrane \\
\hline & Gramicidin S and Tyrocidine & & Cytoplasmic membrane \\
\hline & Clofazimine & & Cytoplasmic membrane \\
\hline & Macrocyclic lactones & Primycin & Membrane function, membrane ATPase? \\
\hline \multicolumn{4}{|l|}{ Bacterial protein } \\
\hline \multirow{2}{*}{\multicolumn{4}{|c|}{$\begin{array}{l}\text { biosynthesis } \\
\text { Bacterial ribosome }\end{array}$}} \\
\hline & & & \\
\hline \multirow[t]{3}{*}{30 S subunit } & Aminoglycosides & Tobramycin & 16S rRNA (A-site) initiation complex/translation \\
\hline & Tetracyclines & Doxycycline & 16S rRNA (A-site) \\
\hline & Macrocyclic peptide & Capreomycin & $16 \mathrm{~S}$ rRNA \\
\hline \multirow[t]{11}{*}{50 S subunit } & Phenyl propanoids & Chloramphenicol & 23S rRNA (peptidyl transferase centre) \\
\hline & \multirow[t]{3}{*}{ Macrolides } & Erythromycin & \multirow[t]{3}{*}{ 23S rRNA (A2058, A2059 in exit tunnel) (translocation) } \\
\hline & & Clarithromycin & \\
\hline & & Roxithromycin & \\
\hline & \multirow[t]{2}{*}{ Ketolides } & Azithromycin & As macrolides plus additional binding sites \\
\hline & & Telithromycin & \\
\hline & Lincosamides & Clindamycin & $23 \mathrm{~S}$ rRNA (A- and P-sites of peptidyl transferase centre) \\
\hline & Oxazolidinones & Linezolid & $23 \mathrm{~S}$ rRNA \\
\hline & \multirow[t]{2}{*}{ Streptogramins } & Quinupristin & As macrolides \\
\hline & & Dalfopristin & Binds close to quinupristin (peptidyl transferase centre) \\
\hline & Pleuromutilins & Retapamulin & 23S rRNA (peptidyl transferase centre) \\
\hline Elongation factors & Steroids & Fusidic acid & Elongation factor G (EF-G)-GTP/GDP complex \\
\hline \multirow{3}{*}{$\begin{array}{l}\text { DNA } \\
\text { Synthesis (replication and } \\
\text { repair, transcription) }\end{array}$} & \multirow[t]{2}{*}{ (Fluoro)quinolones } & \multirow[t]{2}{*}{ Ciprofloxacin } & A-subunit of DNA gyrase in E. coli, ParC of topoisomerase \\
\hline & & & IV in S. pneumoniae \\
\hline & Coumermycins & Novobiocin & ATP-binding site of GyrB \\
\hline RNA & \multirow[t]{3}{*}{ Ansamycins: Rifamycins } & Rifampicin & \multirow[t]{3}{*}{$\beta$-subunit of RNA polymerase } \\
\hline \multirow[t]{2}{*}{ Synthesis (transcription) } & & Rifabutin & \\
\hline & & Rifapentin & \\
\hline tRNA synthesis & Pseudomonic acids & Mupirocin & Isoleucyl-tRNA synthetase \\
\hline
\end{tabular}


continued Table 1. Targets used by commercialised antimicrobial agents (adapted from reference 38). Antimicrobial classes highlighted in bold are discussed.

\begin{tabular}{|c|c|c|c|}
\hline \multirow[t]{4}{*}{ Folate biosynthesis } & $\begin{array}{l}\text { Diaminopyrimidines } \\
\text { (antifolates) }\end{array}$ & Trimethoprim & Dihydrofolate reductase \\
\hline & Sulphonamides & Sulfamethoxazole & Dihydropteroate synthase \\
\hline & & p-aminosalicylic acid & Dihydropteroate synthase other? \\
\hline & Sulphones & Dapsone & Dihydropteroate synthase \\
\hline \multirow{7}{*}{$\begin{array}{l}\text { Other targets } \\
\text { Fatty acid and mycolic } \\
\text { acid biosynthesis }\end{array}$} & Isoniazid (INH) & & $\begin{array}{l}\mathrm{NADH} \text {-dependent enoyl-ACP reductase (activation by } \\
\text { catalase peroxidase, KatG) and DHFR }\end{array}$ \\
\hline & $\begin{array}{l}\text { Ethionamide and } \\
\text { prothionamide }\end{array}$ & & Same as INH (after activation by EtaA) \\
\hline & Thiourea & |soxyl & Membrane-bound $\Delta$ 9-desaturase \\
\hline & Thiacetazone & & Same as INH (after activation by EtaA) \\
\hline & Pyrazinamide & & $\begin{array}{l}\text { Membrane energetics (after conversion to pirazinoic acid } \\
\text { by pyrazinamidase) }\end{array}$ \\
\hline & Ethambutol & & Arabinosyl transferases EmbA, EmbB, EmbC \\
\hline & Triclosan & & Enoyl reductase, Fabl (InhA) \\
\hline \multirow{4}{*}{$\begin{array}{l}\text { Agents exerting } \\
\text { pleiotropic or unknown } \\
\text { effect }\end{array}$} & Nitrofurans & Nitrofurantoin & Multiple sites, esp. ribosomal proteins \\
\hline & Nitroimidazoles & Metronidazole & $\begin{array}{l}\text { Pyruvate-ferredoxin oxidoreductase, DNA (after activation } \\
\text { to reactive species) }\end{array}$ \\
\hline & Nitroquinolines & Nitroxoline & Membrane function \\
\hline & Acridines & Ethacridine & DNA function \\
\hline
\end{tabular}

Table 2. Resistance mechanisms to the main antibacterial classes.

\begin{tabular}{|c|c|c|c|}
\hline $\begin{array}{l}\text { Antibacterial } \\
\text { class }\end{array}$ & $\begin{array}{l}\text { Resistance } \\
\text { mechanism }\end{array}$ & More specific & Mode of action \\
\hline$\beta$-lactam & Inactivation & $\beta$-lactamases (ESBL) & Hydrolysis of beta-lactam ring \\
\hline \multirow[t]{6}{*}{ antibiotics } & Target site alteration & Production of low-affinity PBPs (e.g. mecA) & \\
\hline & & Mutations in endogenous PBPs & \\
\hline & & Overproduction of PBPs & \\
\hline & & Outer membrane proteins & \\
\hline & Impermeability & MexAB-OprM & Reduced levels of antibiotic in the cell \\
\hline & Efflux & & Reduced levels of antibiotic in the cell \\
\hline \multirow[t]{3}{*}{ Glycopeptides } & Target site alteration & Synthesis of modified PG precursors ending in & Decreased affinity \\
\hline & & D-Ala-D-Lac/Ser (VanA, VanB) & \\
\hline & Impermeability & Sequestration of antibiotic & $\begin{array}{l}\text { Thicker wall synthesized: more non-specific } \\
\text { binding and reduced levels of antibiotic in the cell }\end{array}$ \\
\hline \multirow[t]{3}{*}{ Quinolones } & Target site alteration & $\begin{array}{l}\text { Mutations in DNA gyrase and topoisomerase IV } \\
\text { (QRDR) }\end{array}$ & Decreased affinity \\
\hline & Impermeability & & Reduced levels of antibiotic in the cell \\
\hline & Efflux & NorA, PmrA, EmeA & Reduced levels of antibiotic in the cell \\
\hline \multirow[t]{3}{*}{ Rifampicin } & Inactivation & $\begin{array}{l}\text { Phosphorylation, ADP-ribosyl group transfer, } \\
\text { glycosylation, oxidationi }\end{array}$ & Interference with binding to RNA polymerase \\
\hline & & Mutation in rpoB & Decreased affinity \\
\hline & Target site alteration & & \\
\hline
\end{tabular}


Table 2. Resistance mechanisms to the main antibacterial classes.

\begin{tabular}{|c|c|c|c|}
\hline \multirow[t]{2}{*}{ Aminoglycosides } & 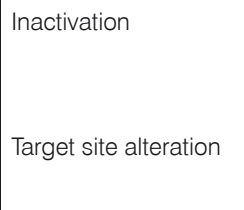 & $\begin{array}{l}\text { Covalent modification: acylation (AAC), } \\
\text { phosphorylation (APH), nucleotidylation (ANT) } \\
\text { Posttranscriptional 16S rRNA methylation ( } r m t A \text {, } \\
\text { rmtB, armA) } \\
\text { Point mutations in rrs (16S rRNA) and rpsL (S12) }\end{array}$ & Decreased affinity \\
\hline & $\begin{array}{l}\text { Impermeability } \\
\text { Efflux }\end{array}$ & & $\begin{array}{l}\text { Reduced levels of antibiotic in the cell } \\
\text { Reduced levels of antibiotic in the cell }\end{array}$ \\
\hline Tetracyclines & $\begin{array}{l}\text { Impermeability } \\
\text { Efflux } \\
\text { Inactivation } \\
\text { Ribosomal protection } \\
\text { systems }\end{array}$ & $\begin{array}{l}\text { Oxidation }(\operatorname{tet}(\mathrm{X})) \\
\operatorname{tet}(\mathrm{M}), \operatorname{tet}(\mathrm{O}), \operatorname{tet}(\mathrm{Q})\end{array}$ & $\begin{array}{l}\text { Reduced levels of antibiotic in the cell } \\
\text { Reduced levels of antibiotic in the cell } \\
\text { Unstable product } \\
\text { Dissociation antibiotic-target interaction }\end{array}$ \\
\hline Chloramphenicol & Inactivation & Acetylation (CAT) & Decreased affinity \\
\hline \multirow[t]{2}{*}{ Macrolides } & Inactivation & $\begin{array}{l}\text { Posttranscriptional 23S rRNA (di)methylation } \\
\left(\mathrm{MLS}_{\mathrm{B}} \text { resistance!) }\right. \\
\text { Hydrolysis (ereA, ereB), phosphorylation (mphA, } \\
m p h B, m p h C) \text {, glycosylation }(\mathrm{mtg})\end{array}$ & Decreased affinity \\
\hline & $\begin{array}{l}\text { Impermeability } \\
\text { Efflux }\end{array}$ & mef & $\begin{array}{l}\text { Reduced levels of antibiotic in the cell } \\
\text { Reduced levels of antibiotic in the cell }\end{array}$ \\
\hline \multirow[t]{2}{*}{ Lincosamides } & Target site alteration & $\begin{array}{l}\mathrm{MLS}_{\mathrm{B}} \text { resistance } \\
\text { Posttranscriptional } 23 \mathrm{~S} \text { rRNA methylation (cfr) } \\
\text { (PhLOPSA) } \\
\text { Mutations in } 23 \mathrm{~S} \text { rRNA and L4/L22 }\end{array}$ & Decreased affinity \\
\hline & $\begin{array}{l}\text { Inactivation } \\
\text { Efflux }\end{array}$ & Nucleotidyl group transfer $\left(\operatorname{lin} A, \operatorname{lin} A^{\prime}, \operatorname{lin} B\right)$ & $\begin{array}{l}\text { Decreased affinity } \\
\text { Reduced levels of antibiotic in the cell }\end{array}$ \\
\hline \multirow[t]{3}{*}{ Streptogramins } & Target site alteration & $\begin{array}{l}\text { MLS }_{B} \text { resistance } \\
\text { Mutations in L4/L22 (rp/ })\end{array}$ & Decreased affinity \\
\hline & Inactivation & $\begin{array}{l}\text { Type A: acetylation }(V A T), \text { reduction } \\
\text { Type B: Iyase }(\operatorname{vgb}(A), \operatorname{vg} b(B))\end{array}$ & Decreased affinity \\
\hline & $\begin{array}{l}\text { Efflux } \\
\text { Impermeability }\end{array}$ & $\begin{array}{l}\text { Type A: vga(A), vga(B) } \\
\text { Type B: } m s r A, m s r S A, m s r B, m s r C \\
\text { Impermeable outer membrane of Gram-negative } \\
\text { bacteria (intrinsic) }\end{array}$ & Reduced levels of antibiotic in the cell \\
\hline Oxazolidinones & Target site alteration & $\begin{array}{l}\text { Mutations in } 23 \mathrm{~S} \text { rRNA and L4 or } \\
\text { Posttranscriptional } 23 \mathrm{~S} \text { rRNA methylation }\end{array}$ & Decreased affinity \\
\hline Trimethoprim & Target site alteration & Mutations in dihydrofolate reductase & Decreased affinity \\
\hline Polymyxin & Target site alteration & Lipid A modification & Interaction blocked \\
\hline Daptomycin & Impermeability & $\begin{array}{l}\text { Impermeable outer membrane of Gram-negative } \\
\text { bacteria (intrinsic) } \\
\text { Thicker cell wall }\end{array}$ & Reduced levels of antibiotic in the cell \\
\hline
\end{tabular}


Figure 4. The cell wall (peptidoglycan matrix) surrounding bacterial cells is a mesh-like carbohydrate polymer with glycan strands connected by peptide cross-links. Transglycosylases catalyse cross-linking of the glycan strands, and the peptide strands undergo cross-linking by the action of transpeptidases. Transpeptidases are inactivated by $\beta$-lactams and glycopeptides. Glycopeptides also inhibit transglycosylase activity. Interference with cross-linking results in cell lysis and death. M: N-acetylmuramic acid; G: N-acetyl-Dglucosamine.

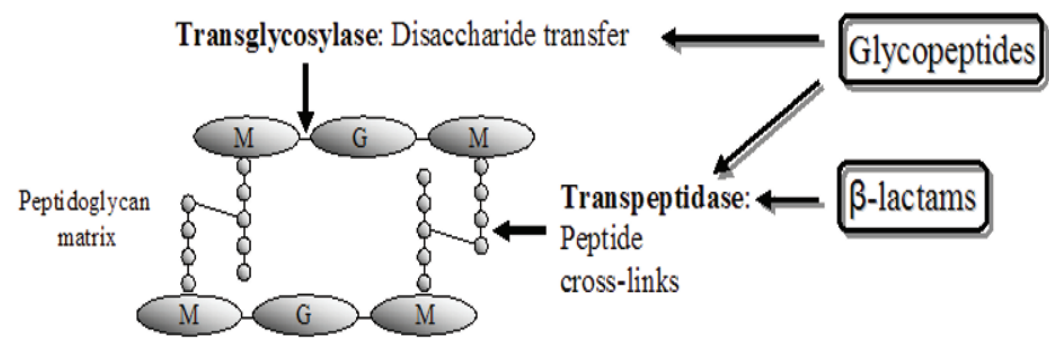

Figure 5. (Fluoro)quinolones and rifamycins interfere with nucleic acid biosynthesis. (Fluoro)quinolones inhibit DNA synthesis by interacting with DNA gyrase and topoisomerase IV resulting in inhibition of normal enzyme activity. Rifamycins act as allosteric inhibitors of the bacterial DNA-dependent RNA polymerase resulting in inhibition of transcription.

\section{DNA/RNA biosynthesis}

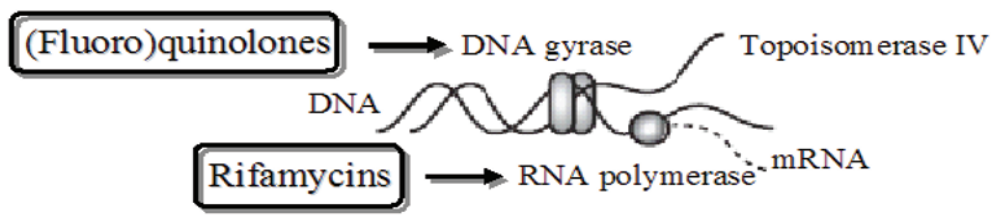

expression is a principle mechanism of Gram-negative resistance [13]. There are four classes of $\beta$-lactamases: three serine-dependent enzyme classes ( $A, C$ and $D)$ and one metal-dependent class (B) [13]. Of particular concern are the enzymes able to target the expanded spectrum $\beta$-lactams, such as the AmpC enzymes, the so-called extended spectrum $\beta$-lactamases (ESBL) and the carbapenemases $[6,12]$.

\section{Target site alteration}

There are several PBP-mediated mechanisms of $\beta$-lactam resistance, including acquisition of a 'new' less-sensitive enzyme, mutation of an endogenous PBP to lessen the reaction with $\beta$-lactams (while maintaining some transpeptidase activity) or upregulation of PBP expression [11]. PBP alteration is a principle mechanism of Gram-positive resistance [13]. The most important example is the acquisition and expression of the mecA gene by $S$. aureus, encoding a new low-affinity PBP, PBP2a (also called PBP2'). This gene is found on a mobile element, the staphylococcal cassette chromosome mec (SCCmec), carrying additional antibiotic (non- $\beta$-lactam) resistance genes [11]. Alterations in or overproduction of other PBPs are also possible [12].

\section{Decreased permeability and increased efflux}

Reduced outer membrane permeability to $\beta$-lactams as a result of porin loss or changes in porin structure can promote resistance to these agents $[12,13]$. A major contribution to antibiotic resistance in Gram-negative species is the presence of broad-specificity drug-efflux pumps. One of the best characterized of these is the drug efflux system MexAB-OprM of $P$. aeruginosa [11-13].

To overcome resistance, semisynthetic $\beta$-lactamase resistant $\beta$-lactams were developed $[3,12]$. $\beta$-Lactamase susceptible $\beta$-lactams can be co-administered with $\beta$-lactamase inhibitors, such as clavulanic acid, sulbactam and tazobactam $[3,12]$. A number of $\beta$-lactam compounds that bind strongly to low-affinity PBPs have been designed as well as agents that potentiate the activity of existing $\beta$-lactams against low-affinity PBP-producing organisms [8,12]. Dual action hybrid antimicrobials were designed by fusing $\beta$-lactams to other antimicrobials, harnessing the enzymatic action of $\beta$-lactamases, which on their turn release the second antimicrobial $[3,12,14]$.

\subsubsection{Glycopeptides}

Glycopeptides bind highly specific, non-covalently to the D-Ala ${ }_{4}$-D-Ala ${ }_{5}$ termini of the UDP-muramylpentapeptide peptidoglycan precursors. Through this binding, the bound glycopeptide acts as a steric impediment. The substrates are kept away from transglycosidase (chain elongation) and transpeptidase (crosslinking). This substrate sequestration leads to the failure of peptidoglycan cross-links, making the cell wall susceptible to osmolysis $[2,8,9,15,16]$. Whereas binding to the D-Ala - D-Ala 5 peptide motif is crucial for antimicrobial activity, the mode of action is still 
more sophisticated and dimerization and membrane anchoring have been suggested [16]. This stabilises the binding to the new depsipeptide or facilitates a new mechanism of action, namely the active site inhibition of transglycosylase activity [5].

\section{Target site alteration and impermeability}

The most frequent cause of resistance in enterococci (vancyomycin resistant enterococci, VRE) is the acquisition of one of two related gene clusters, vanA or vanB, located on transposable elements [14]. This results in synthesis of peptidoglycan by an alternative pathway, which produces modified peptidoglycan precursors ending in D-Ala 4 -D-Lac or D-Ala $_{4}-\mathrm{D}_{5}-\mathrm{Ser}_{5}$ instead of D-Ala 4 -D-Ala ${ }_{5}$ and concomitantly eliminates precursors ending in D-Ala ${ }_{4}-\mathrm{D}^{-} \mathrm{Ala}_{5}$ [14]. This causes loss of binding affinity $[3,9]$. The VanA phenotype shows resistance to both glycopeptide drugs, vancomycin and teicoplanin, while the VanB phenotype is resistant to vancomycin, but remains susceptible to teicoplanin [9].

Sequestration of the agent in a modified wall structure or in the medium has been noticed. Vancomycin resistant Staphylococcus aureus (VRSA) strains typically generate multilayered, thickened cell walls as if more sites for stoichiometric binding of drugs are the cause of reduced susceptibility [8-10].

The second-generation semi-synthetic lipoglycopeptides oritavancin, telavancin and dalbavancin as well as chlorobiphenyl vancomycin analogues retain activity against these resistant strains $[8,9]$.

\subsection{Inhibitors of nucleic acid biosynthesis}

Two classes of antimicrobials are known to interfere with nucleic acid biosynthesis, (fluoro)quinolones and rifamycins (Figure 5).

\subsubsection{Quinolones}

In order to fit inside the bacterium, the DNA is negatively supercoiled and is arranged around an RNA core. The topological stress during transcription or DNA replication is relieved and the positive supercoils are removed by a type II topoisomerase, known as DNA gyrase, which makes double-stranded breaks in the DNA and reduces the linking number by two [17]. Following DNA synthesis, the daughter chromosomes are unlinked by an other type II topoisomerase, topoisomerase IV, in a process called decatenation $[2,17]$.

(Fluoro)quinolones inhibit DNA synthesis and at higher concentrations they also inhibit RNA synthesis [18]. They interact with the complexes formed between DNA and the DNA gyrase or topoisomerase IV creating conformational changes that result in inhibition of the normal enzyme activity. DNA gyrase seems to be the primary target for Gram-negative organisms, while topoisomerase IV is the primary target in Gram-positive organisms $[2,6,19]$. There are two steps to quinolone action: formation of bacteriostatic drug-enzyme-DNA complexes, followed by the release of lethal doublestranded DNA breaks $[8,18-20]$.

\section{Target site alteration and reduced uptake}

Resistance is mediated chiefly through stepwise (spontaneous) target mutations in the genes encoding subunits of DNAgyrase (primarily gyrA) or topoisomerase IV (primarily parC) or both. The resistant mutations cluster in specific highly conserved regions of the genes centered around the active site, called the quinolone resistance determining region (QRDR). These mutations alter the structure of the quinolone binding site leading to reduced drug affinity for the modified enzyme-DNA complex [6,8,17-19,21].

Other mechanisms that contribute to decreased sensitivity to these drugs are energy-dependent efflux and reduced permeability due to reduced amounts of general diffusion porins [6]. The efflux pumps include NorA (S. aureus), PmrA (S. pneumoniae) and EmeA $[17,21,22]$. This increases the likelihood of further selection of resistance by target mutation mechanisms $[19,20]$.

The design of compounds with a 'balanced' activity and affinity against both primary topoisomerase targets, such as 8-methoxy quinolones, fourth generation quinolones and non-fluorinated quinolones (NFQs), makes the selection of concomitant genetic resistance to both targets and, consequently, emergence of de novo resistance, less likely to happen $[18,20]$. The therapeutic use of efflux inhibitors may be a strategy to lower fluoroquinolone resistance [17].

\subsubsection{Rifamycins, RNA transcription inhibitors}

Transcription is an essential process for decoding genetic information from DNA to mRNA in all organisms. The RNA polymerase of bacteria, composed of different subunits with a stoichiometry of $\alpha_{2} \beta \beta^{\prime} \omega$ to form the core enzyme, catalyses transcription [2].

Rifampicin, important in combination therapy in the treatment of Mycobacterium tuberculosis infections, inhibits bacterial DNA-dependent RNA polymerase by binding to the $\beta$-subunit of the enzyme, encoded by $r p o B$, at an allosteric site. It apparently blocks the entry of the first nucleotide, which is necessary to activate the polymerase, thereby blocking mRNA synthesis $[2,6,8,21]$. 
Figure 6. The process of protein biosynthesis can be inhibited by compounds targeting the $30 \mathrm{~S}$ or the $50 \mathrm{~S}$ subunit of the bacterial ribosome.

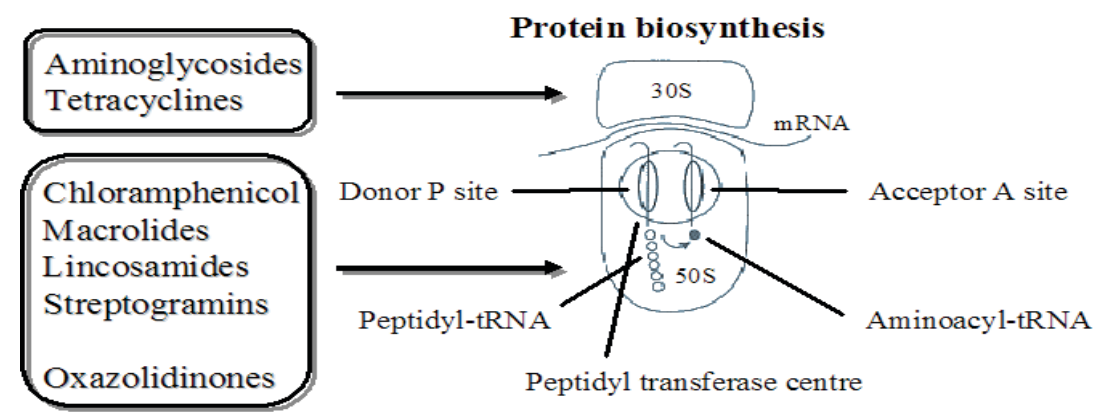

Antibiotic inactivation

A number of mechanisms (uncharacterised kinase, ADP-ribosyl transferase (ARR), glycosylation, monooxygenase) can modify the hydroxyl group at position 23 of rifampicin and presumably interfere with binding to RNA polymerase [3,21].

Target site alteration

Resistance due to modification of the $\beta$-subunit of the enzyme through chromosomal mutations in rpoB in $M$. tuberculosis arises with a high frequency [21].

\subsection{Inhibitors of protein biosynthesis}

Protein biosynthesis is catalysed by ribosomes and cytoplasmic factors. The bacterial $70 \mathrm{~S}$ ribosome is composed of two ribonucleoprotein subunits, the 305 and $50 S$ subunits [2]. The smaller $30 S$ subunit is made up of $16 \mathrm{~S}$ rRNA and about 21 ribosomal proteins (S1 to S21), while the larger 50S subunit consists of two RNA molecules, 5S rRNA and 23S rRNA and over 36 ribosomal proteins ( $\mathrm{L} 1$ to $\mathrm{L} 36$ ). The catalytic ribozyme domain of the $23 \mathrm{~S}$ rRNA possesses peptidyl transferase activity and catalyses peptide bond formation [23,24]. Antimicrobials inhibit protein biosynthesis by targeting the $30 \mathrm{~S}$ or $50 \mathrm{~S}$ subunit of the bacterial ribosome (Figure 6).

\subsubsection{Inhibitors of 30 S subunit \\ 3.3.1.1 Aminoglycosides}

Aminoglycosides interact with the conserved sequences of the 16S rRNA of the $30 S$ subunit near the A site through hydrogen bonds. They cause misreading and premature termination of translation of mRNA. The aberrant proteins may be inserted into the cell membrane leading to altered permeability and further stimulation of aminoglycoside transport $[2,3,6,8,25]$.

\section{Antibiotic inactivation}

Inactivation by covalent modification of the key hydroxyl and amine groups on the aminoglycoside antibiotics is the most significant form of acquired resistance in both Gram-negative and Gram-positive bacteria $[3,25,26]$. There are three types of aminoglycosidemodifying enzymes (AMEs), each with many variants: aminoglycoside acetyltransferases (AAC), aminoglycoside phosphotransferases (kinases) (APH) and aminoglycoside adenylyltransferases (ANT) $[2,6,21]$.

\section{Target site alteration}

Many aminoglycoside producing organisms express rRNA methylases (aminoglycoside resistance family of methyltransferases), whichmodify the 16SrRNAmolecule at specific positions critical for the tight binding of the drug. This is highlighted by the finding of the $r m t A, r m t B$ and armA genes [26,27] causing a posttranscriptional 16S rRNA methylation. Aminoglycoside resistance can also occur by point mutations in the rrs gene, encoding the 16S rRNA of the $30 S$ subunit, or by mutations in the $r p s L$ gene, encoding the $30 \mathrm{~S}$ ribosomal protein $\mathrm{S} 12$. These ribosomal mutations are clinically relevant only for streptomycin in M. tuberculosis [8,26].

\section{Decreased permeability and increased efflux}

Finally, aminoglycoside concentrations can be decreased inside a target cell by reduction of drug uptake, activation of drug efflux pump or both [25,26].

The most successful approach to combat resistance is by development of aminoglycosides that lack sites of inactivation, as exemplified by amikacin, which is protected from attack by steric hindrance due to the presence of a side chain [25]. The second approach is the design of inhibitors of the three classes of aminoglycoside-modifying enzymes. Inhibitor design can be targeted at the aminoglycoside, cofactor binding sites or both [25].

\subsubsection{Tetracyclines}

Tetracyclines can be divided into two types based on their mode of action [28]. Typical tetracyclines, such as tetracycline, chlortetracycline, doxycycline or minocycline, act upon the conserved sequences 
of the 16S rRNA of the $30 \mathrm{~S}$ ribosomal subunit to prevent binding of tRNA to the A site $[2,6]$. Some other tetracycline derivatives, such as chelocardin, thiatetracycline, anhydrotetracycline, have been shown to act by inserting into the cytoplasmic membrane [28]. Thirty-eight acquired genetically mobile tetracycline (tet) and oxitetracycline (otr) resistance $\left(\mathrm{Tc}^{r}\right)$ genes are known, including genes coding for energy-dependent efflux proteins, ribosomal protection proteins and tetracycline inactivating enzymes [29].

Alteration of porin proteins, e.g. OmpF, or other outer membrane proteins limits the diffusion of tetracycline into the periplasm in Gram-negative bacteria [28]. About $60 \%$ of all tet and otr genes code for energy-dependent membrane-associated transporters belonging to the MFS, which export tetracycline out of the cell at a rate equal to or greater than its uptake $[28,29]$. On the other hand, ribosomal protection proteins promote GTPdependent release of tetracyclines from the ribosomal A site leading to dissociation of the antibiotic-target interaction $[29,30]$. Three classes of ribosome protection resistance genes have been described, tet $(\mathrm{M}), \operatorname{tet}(\mathrm{O})$ and tet( $\mathrm{Q})$ genes [28]. Resistance can also arise by point mutation in ribosomal RNA [30].

Finally, the tet( $\mathrm{X})$ gene encodes an NADPHrequiring oxidoreductase, which oxidizes tetracycline antibiotics. The antibiotic undergoes non-enzymatic rearrangement into unstable products that polymerise into a black product after several hours [3,28,29].

A new generation of tetracyclines, the 9-glycinyltetracyclines or glycylcyclines (tigecycline, 9-t-butylglycylamido-minocycline) have been developed. Glycylcyclines have a higher binding affinity for ribosomes than earlier tetracyclines. Furthermore, the Tet efflux proteins fail to recognise glycylcyclines or are unable to transfer glycylcyclines [30]. A number of tetracycline efflux pump inhibitors have been discovered that might be used in combination with earlier tetracyclines.

\subsubsection{Inhibitors of 50 S subunit}

\subsubsection{Chloramphenicol}

Chloramphenicol interacts with the conserved sequences of the peptidyl transferase cavity of the 23S rRNA of the 50 S subunit. It inhibits protein synthesis by preventing binding of tRNA to the A site of the ribosome. It interacts with various nucleotides of the peptidyl transferase cavity of the 23S rRNA through hydrogen bonds [2,23].

\section{Antibiotic inactivation}

The inactivation of chloramphenicol is accomplished by the chloramphenicol acetyltransferases (CAT) by transferring the acetyl group from acetyl CoA, resulting in a lower affinity of the antibiotic for the rRNA [3]. Hereto, florfenicol was developed to overcome CAT-mediated resistance.

\subsubsection{Macrolides}

Macrolides affect the early stage of protein synthesis, namely translocation, by targeting the conserved sequences of the peptidyl transferase centre of the 235 rRNA of the $50 \mathrm{~S}$ ribosomal subunit $[2,6]$. This results in a premature detachment of incomplete peptide chains [23]. Although compounds of considerable structural variety, macrolides, lincosamides and streptogramins $B$ $\left(\mathrm{MLS}_{\mathrm{B}}\right.$ antibiotics) show a similar mechanistic action.

\section{Target site alteration}

Any discussion of mechanisms of resistance to macrolide antimicrobials must include the lincosamide and streptogramin B families as well. This type of cross-resistance has therefore been referred to as MLS $_{B}$ resistance $[31,32]$ and is generally the result of target site alteration. The latter results from a post-transcriptional modification of the 23S rRNA component of the $50 \mathrm{~S}$ ribosomal subunit involving methylation or dimethylation of A2058 (E. coli numbering) in the peptidyl transferase functional domain. This is catalysed by adenine-specific $\mathrm{N}$-methyltransferases (methylases, MTases) specified by the erm class of genes, frequently plasmid encoded $[8,32]$. This modification reduces the affinity of the rRNA for the antimicrobials but does not interfere with protein biosynthesis [2]. Mutations in 23S rRNA close to the sites of methylation can also lead to macrolide resistance [8,32]. In addition to multiple mutations in the 23S rRNA, mutations in the L4 and L22 50 S ribosomal proteins have also been seen $[8,32]$.

\section{Antibiotic inactivation}

Macrolides can also be inactivated by specific enzymes inside the cell, such as proteins that cleave the macrocycle ester, encoded by ere $A$ and ere $B$ genes. Phosphorylation by MPHs (macrolide kinases) encoded by $m p h A$ and $m p h B$ from $E$. coli and mphBM (mphC) from $S$. aureus and macrolide glycosylation by the product of the $m t g$ gene [3,31] are also possible.

\section{Decreased permeability and increased efflux}

Finally, macrolide entrance into bacterial cell can be prevented by changes in the permeability of the membrane or the cell wall. The active extrusion of antimicrobials from the bacterial cell by the action of efflux pumps, encoded by mef genes, has also been observed [21,31].

The ketolide telithromycin retains activity against isolates resistant by target modification [8]. Activity of 
the existing antimicrobial drugs can also be restored by the design of inhibitors of the Erm MTases [31].

\subsubsection{Lincosamides}

Lincosamides interact with the conserved sequences of the 23S rRNA of the $50 \mathrm{~S}$ subunit [2]. They act by affecting the process of peptide chain initiation and may also stimulate dissocation of peptidyl-tRNA from ribosomes. In contrast with macrolides, lincosamides are direct peptidyltransferase inhibitors [33].

\section{Target site alteration}

The main type of resistance is the $\mathrm{MLS}_{B}$ resistance [33]. Recently, methylation of 23S rRNA at A2503 by the cfr gene product has been seen. Cfr causes resistance by inhibiting ribose methylation at nucleotide C2498. The phenotype was named PhLOPSA [33]. Mutations in the 23S rRNA and in L4 and L22 ribosomal protein genes likewise have been found [33].

\section{Antibiotic inactivation and efflux}

As inactivation mechanism, three lincosamide O-nucleotidyltransferase genes, $\operatorname{lin} A$, $\operatorname{lin} A$ ' and $\operatorname{lin} B$ $[3,33]$ have been characterized. Alternatively, efflux of the antibiotic is the main resistance mechanism in Gram-negative bacteria [33].

No specific mechanisms to overcome the ever increasing resistance have been developed, beside the use of combinations of different antibiotics or of antibiotics with non-antibiotic antimicrobials [33].

\subsubsection{Streptogramins}

Streptogramins act by binding to the conserved sequences of the 23S rRNA of the 50 S subunit and by interfering with peptidyltransferase activity [2,9]. Type A streptogramins block the substrate site of the peptidyl transferase centre, thus preventing the earliest event of elongation [23]. Type B streptogramins block peptide bond synthesis and cause a premature release of incomplete peptide chains [23]. The synergism between types $A$ and $B$ streptogramins is due to induction by type $A$ streptogramins of conformational changes in ribosomes that significantly increase the ribosome affinity for type B streptogramins [23,24].

Resistance to streptogramin combinations requires resistance specifically to the $\mathrm{S}_{\mathrm{A}}$ component, but it is augmented by the presence of mechanisms conferring $\mathrm{S}_{\mathrm{B}}$ resistance [21].

\section{Target site alteration}

The main type of resistance is the $M_{L} S_{B}$ resistance. Type A streptogramins are not affected by this altered residue and the efficacy of the synergistic combination is thus retained [21,24]. Also, modification of 23 rRNA ribosomal proteins, such as ribosomal protein $L 4$, due to point mutations and small deletions or insertions has been described. Low-level resistance has been reported resulting from mutations in $r p / V$, which encodes ribosomal protein L22 [21].

\section{Antibiotic inactivation}

The streptogramin acetyltransferases (VATs) inactivate the type $A$ streptogramins by $O$-acetylation. Five acetyltransferases, encoded by $\operatorname{vat}(A), \operatorname{vat}(B)$ and $\operatorname{vat}(C)$ and by $\operatorname{vat}(D)$ and $\operatorname{vat}(E)$, have been seen $[21,24]$. The producer of the type $A$ streptogramin virginiamycin $M_{1}$ protects itself by reducing a critical ketone group, thereby generating an inactive compound. This reduction is NADPH-dependent and regiospecific [3]. Specific resistance to type $B$ streptogramins is mediated by lyases, encoded by $\operatorname{vg} b(\mathrm{~A})$ and $\operatorname{vg} b(\mathrm{~B})$, which inactivate the compounds via an elimination mechanism $[3,21,24]$.

\section{Decreased uptake and increased efflux}

Alternatively, streptogramin uptake into the periplasm is impaired among most Gram-negative organisms owing to the impermeable Gram-negative outer membrane (intrinsic resistance) [24]. Active efflux of type A streptogramins is due to ATP-binding cassette proteins encoded by plasmid-borne $v g a(A)$ and $v g a(B)$ genes [21]. Efflux of type $B$ streptogramins is due to the presence of another ATP-binding transporter encoded by the $m s r A, m s r S A, m s r B$ and $m s r C$ genes [24].

\subsubsection{Oxazolidinones}

Oxazolidinones inhibit formation of the 705 initiation complex by binding to the $\mathrm{P}$ site at the $50 \mathrm{~S}$ ribosomal subunit near to the interface with the $30 S$ subunit, thereby blocking the first peptide-bond forming step [9]. If the $70 S$ initiation complex is already formed, they inhibit translocation of peptidyl-tRNA from the A site to the $P$ site during formation of the peptide bond $[8,34]$. Recently it was shown that they also inhibit fMet-tRNA binding to the $\mathrm{P}$ site [34].

As the action mechanism of oxazolidinones is unique, no cross-resistance between oxazolidinones and other protein synthesis inhibitors has been observed [34].

\section{Target site alteration}

Resistance arises by spontaneous mutations in chromosomal genes encoding 23S rRNA, resulting in decreased affinity for binding, or in protein $L 4[8,21,34]$. Also posttranscriptional modification of the target site is a possible cause of resistance. Linezolid resistance is determined by the presence of the cfr gene. Cfr 
Figure 7. Sulphonamides and trimethoprim inhibit distinct steps in folate metabolism.

\section{Folate metabolism}

Pteridine (dihydropteroate diphosphate) $+\mathrm{p}$-amin obenzoic acid (PABA)

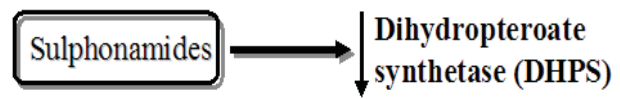

Dihydropteroic acid (DHP)

I Dihydrofolate synthetase (DHFS)

Dihydrofolic acid $\left(\mathrm{H}_{2}\right.$ folate, DHF)

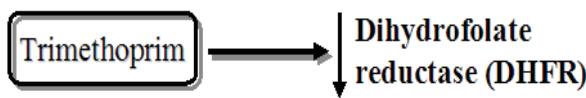

Tetrahydrofolic acid $\left(\mathrm{H}_{4}\right.$ folate, THF)

methyltransferase modifies adenosine at position 2503 in $23 \mathrm{~S}$ rRNA. The natural function of cfr likely involves protection against natural antibiotics whose site of action overlaps that of linezolid [35].

\subsection{Miscellaneous targets}

\subsubsection{Folic acid metabolism: Sulphonamides and Trimethoprim}

Each of these drugs inhibits distinct steps in folic acid metabolism (Figure 7). A combination of sulpha drugs and trimethoprim acting at distinct steps on the same biosynthetic pathway shows synergy and a reduced mutation rate for resistance [2]. Sulphonamides inhibit dihydropteroate synthase in a competitive manner with higher affinity for the enzyme than the natural substrate, p-aminobenzoic acid. Agents such as trimethoprim act at a later stage of folic acid synthesis and inhibit the enzyme dihydrofolate reductase [2].

\section{Target site alteration}

Mutations in the $d(h) f r$ gene producing single amino acid substitution in the dihydrofolate reductase are responsible for trimethoprim resistance. Changes in both the promoter and coding regions of the dhfr gene have been found [8]. Overexpression or metabolic bypass of the target has also been observed.

\subsubsection{Cell membrane disruptors}

\subsubsection{Polymyxin antibiotics}

Cationic cyclic peptides with a fatty acid chain attached to the peptide, such as polymyxins, attack the cytoplasmic membrane of Gram-positive and Gram-negative bacteria and the outer membrane of Gram-negative bacteria. They bind to phospholipids in the cytoplasmic membrane, causing loss of membrane integrity, leakage of cytoplasmic contents and finally cell death $[2,8]$. The key initial interaction between the polymyxins and lipopolysaccharides can be blocked by modification of the phosphate esters linked to the diglucosamine components of lipid A [8].

\subsubsection{Lipopeptides: Daptomycin}

Daptomycin has a unique mode of action and involves a calcium-dependent insertion of the lipid side chain into the Gram-positive cell membrane. After this, several molecules come together to form oligomers that disrupt the cell membrane without entering the cytoplasm of the cell. This ion-conduction structure results in potassium efflux and associated membrane depolarisation. This disruption of the bacterial cell membrane function also appears to trigger inhibition of DNA, RNA and protein synthesis resulting in cell death [9,36,37]. The synthesis of lipotheichoic acid, found in Gram-positive organisms, is also inhibited by daptomycin [36]. Due to its unique mode of action, there is generally no crossresistance [37]. Spontaneous acquisition of resistance to daptomycin occurs rarely [36].

\section{Impermeability}

The failure to cross the outer membrane of Gram-negative bacteria to reach the inner cell membrane target is likely to explain the lack of daptomycin activity against Gramnegative bacteria (intrinsic resistance) [37]. Correlation with vancomycin and daptomycin resistance linked to the thickness of the cell wall suggests that prior use of vancomycin may predispose to decreased daptomycin susceptibility [36]. 
4.

Conclusion: continuous
development of novel
antibacterials is mandatory

This overview has given insight in the many therapeutic possibilities that exist for treatment of bacterial infections and in the continuous battle between resistance development and overriding mechanisms. Therefore, to prevent the emergence and dissemination of resistant bacteria, continuing efforts to develop new antibacterial agents are warranted. Although this is not an easy

\section{References}

[1] Cohen M.L., Changing patterns of infectious disease, Nature, 2000, 406, 762-767

[2] Yoneyama H., Katsumata R., Antibiotic resistance in bacteria and its future for novel antibiotic development, Biosci. Biotechnol. Biochem., 2006, 70, 1060-1075

[3] Wright G.D., Bacterial resistance to antibiotics: enzymatic degradation and modification, Adv. Drug Deliv. Rev., 2005, 57, 1451-1470

[4] Rachakonda S., Cartee L., Challenges in antimicrobial drug discovery and the potential of nucleoside antibiotics, Curr. Med. Chem., 2004, 11, 775-793

[5] Silver L.L., Novel inhibitors of bacterial cell wall synthesis, Curr. Opin. Microbiol., 2003, 6, 431-438

[6] Wise R., A review of the mechanisms of action and resistance of antimicrobial agents, Can. Respir. J., 1999, 6 Suppl A, 20A-22A

[7] Langton K.P., Henderson P.J., Herbert R.B., Antibiotic resistance: multidrug efflux proteins, a common transport mechanism?, Nat. Prod. Rep., 2005, 22, 439-451

[8] Lambert P.A., Bacterial resistance to antibiotics: modified target sites, Adv. Drug Deliv. Rev., 2005, 57, 1471-1485

[9] Kahne D., Leimkuhler C., Lu W., Walsh C., Glycopeptide and lipoglycopeptide antibiotics, Chem. Rev., 2005, 105, 425-448

[10] Reynolds P.E., Structure, biochemistry and mechanism of action of glycopeptide antibiotics, Eur. J. Clin. Microbiol. Infect. Dis., 1989, 8, 943-950

[11] Wilke M.S., Lovering A.L., Strynadka N.C., Betalactam antibiotic resistance: a current structural perspective, Curr. Opin. Microbiol., 2005, 8, 525-533

[12] Poole K., Resistance to beta-lactam antibiotics, Cell Mol. Life Sci., 2004, 61, 2200-2223

[13] Fisher J.F., Meroueh S.O., Mobashery S., Bacterial assignment, there is still hope and many new avenues are being explored. Indeed, the recent advance in bacterial genomics has changed the antibacterial therapeutic environment from target-poor to target-rich, hence many potential targets are awaiting $[2,4,8,38]$.

\section{Acknowledgements}

K.B. is a research fellow of the Research foundation Flanders (FWO-Vlaanderen).

resistance to beta-lactam antibiotics: compelling opportunism, compelling opportunity, Chem. Rev., 2005, 105, 395-424

[14] Van Bambeke F., Van Laethem Y., Courvalin P., Tulkens P.M., Glycopeptide antibiotics: from conventional molecules to new derivatives, Drugs, 2004, 64, 913-936

[15] Williams D.H., The glycopeptide story--how to kill the deadly 'superbugs', Nat. Prod. Rep., 1996, 13, 469-477

[16] Sussmuth R.D., Vancomycin resistance: small molecule approaches targeting the bacterial cell wall biosynthesis, Chembiochem., 2002, 3, 295-298

[17] Schmitz F.J., Higgins P.G., Mayer S., Fluit A.C., Dalhoff A., Activity of quinolones against Grampositive cocci: mechanisms of drug action and bacterial resistance, Eur. J. Clin. Microbiol. Infect. Dis., 2002, 21, 647-659

[18] Hooper D.C., Mechanisms of action of antimicrobials: focus on fluoroquinolones, Clin. Infect. Dis., 2001, 32 Suppl 1, S9-S15

[19] Higgins P.G., Fluit A.C., Schmitz F.J., Fluoroquinolones: structure and target sites, Curr. Drug Targets., 2003, 4, 181-190

[20] Dougherty T.J., Beaulieu D., Barrett J.F., New quinolones and the impact on resistance, Drug Discov. Today, 2001, 6, 529-536

[21] Woodford N., Biological counterstrike: antibiotic resistance mechanisms of Gram-positive cocci, Clin. Microbiol. Infect., 2005, 11 Suppl 3, 2-21

[22] Eliopoulos G.M., Quinolone resistance mechanisms in pneumococci, Clin. Infect. Dis., 2004, 38 Suppl 4, S350-S356

[23] Vannuffel P., Cocito C., Mechanism of action of streptogramins and macrolides, Drugs, 1996, 51 Suppl 1, 20-30

[24] Johnston N.J., Mukhtar T.A., Wright G.D., 
Streptogramin antibiotics: mode of action and resistance, Curr. Drug Targets., 2002, 3, 335-344

[25] Shahid M., Aminoglycosidic aminocyclitol antibiotics-Awonder, but toxic drugs: Developments and clinical implications, Anti-infect. Agents Med. Chem., 2007, 6, 107-117

[26] Jana S., Deb J.K., Molecular understanding of aminoglycoside action and resistance, Appl. Microbiol. Biotechnol., 2006, 70, 140-150

[27] Kotra L.P., Haddad J., Mobashery S., Aminoglycosides: perspectives on mechanisms of action and resistance and strategies to counter resistance, Antimicrob. Agents Chemother., 2000, 44, 3249-3256

[28] Speer B.S., Shoemaker N.B., Salyers A.A., Bacterial resistance to tetracycline: mechanisms, transfer, and clinical significance, Clin. Microbiol. Rev., 1992, 5, 387-399

[29] Roberts M.C., Update on acquired tetracycline resistance genes, FEMS Microbiol. Lett., 2005, 245, 195-203

[30] Chopra I., Roberts M., Tetracycline antibiotics: mode of action, applications, molecular biology, and epidemiology of bacterial resistance, Microbiol. Mol. Biol. Rev., 2001, 65, 232-260

[31] Maravic G., Macrolide resistance based on the Ermmediated rRNA methylation, Curr. Drug Targets. Infect. Disord., 2004, 4, 193-202
[32] Weisblum B., Erythromycin resistance by ribosome modification, Antimicrob. Agents Chemother., 1995, 39, 577-585

[33] Rezanka T., Spizek J., Sigler K., Medicinal use of lincosamides and microbial resistance to them, Anti-infect. Agents Med. Chem., 2007, 6, 133-144

[34] Bozdogan B., Appelbaum P.C., Oxazolidinones: activity, mode of action, and mechanism of resistance, Int. J. Antimicrob. Agents, 2004, 23, 113-119

[35] Toh S.M., Xiong L., Arias C.A., Villegas M.V., Lolans K., Quinn J., Mankin A.S., Acquisition of a natural resistance gene renders a clinical strain of methicillin-resistant Staphylococcus aureus resistant to the synthetic antibiotic linezolid, Mol. Microbiol., 2007, 64, 1506-1514

[36] Enoch D.A., Bygott J.M., Daly M.L., Karas J.A., Daptomycin, J. Infect., 2007, 55, 205-213

[37] Johnson A., Daptomycin in the treatment of skin, soft-tissue and invasive infections due to Grampositive bacteria, Future. Microbiol., 2006, 1, 255-265

[38] Lange R.P., Locher H.H., Wyss P.C., Then R.L., The targets of currently used antibacterial agents: lessons for drug discovery, Curr. Pharm. Des, 2007, 13, 3140-3154 